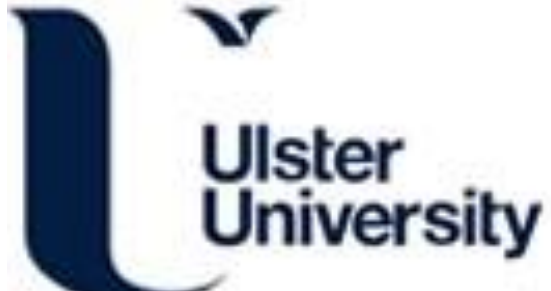

\section{A Flexible and Multipurpose Piezoresistive Strain Sensor Based on Carbonized Phenol Formaldehyde Foam for Human Motion Monitoring}

Wang, L., Xiang, D., Harkin-Jones, E., Zhang, X., Li, Y., Zheng, Y., Zhao, C., \& Wang, P. (2019). A Flexible and Multipurpose Piezoresistive Strain Sensor Based on Carbonized Phenol Formaldehyde Foam for Human Motion Monitoring. Macromolecular Materials and Engineering, 304(12), 1-9. [1900492].

https://doi.org/10.1002/mame.201900492

Link to publication record in Ulster University Research Portal

\section{Published in:}

Macromolecular Materials and Engineering

Publication Status:

Published (in print/issue): 31/12/2019

DOI:

10.1002/mame.201900492

\section{Document Version}

Publisher's PDF, also known as Version of record

\section{General rights}

Copyright for the publications made accessible via Ulster University's Research Portal is retained by the author(s) and / or other copyright owners and it is a condition of accessing these publications that users recognise and abide by the legal requirements associated with these rights.

\section{Take down policy}

The Research Portal is Ulster University's institutional repository that provides access to Ulster's research outputs. Every effort has been made to ensure that content in the Research Portal does not infringe any person's rights, or applicable UK laws. If you discover content in the Research Portal that you believe breaches copyright or violates any law, please contact pure-support@ulster.ac.uk. 


\title{
A Flexible and Multipurpose Piezoresistive Strain Sensor Based on Carbonized Phenol Formaldehyde Foam for Human Motion Monitoring
}

\author{
Lei Wang, Dong Xiang,* Eileen Harkin-Jones, Xuezhong Zhang, Yuntao Li, * \\ Yongfeng Zheng, Chunxia Zhao, and Ping Wang
}

High-performance flexible strain sensors are extensively studied for various applications including healthcare, robots, and human-computer interaction. In most of the reported research, the fabrication of these sensors involves conductive polymer composites containing expensive metallic or carbon nanomaterials. In this study, commercial phenol formaldehyde foam (PFF) is carbonized by a simple high-temperature pyrolysis treatment and encapsulated by polydimethylsiloxane (PDMS) to fabricate a flexible and multipurpose piezoresistive strain sensor. The as-fabricated PDMS-cPFF strain sensor is capable of detecting various strain modes, including tension, compression, and three-point bending. Furthermore, the sensor exhibits a high sensitivity with a gauge factor (GF) of $-\mathbf{2 0 . 5}$ under tension and stable signal responses in a frequency range of $0.01-0.5 \mathrm{~Hz}$. The sensor is also capable of accurately monitoring a subtle bending strain of $0.05 \%$. In addition, the sensor shows excellent durability in cyclic loading/unloading tests up to 1000 cycles. The applications of this strain sensor in both large- (finger bending and neck movement) and small-scale human motion monitoring (facial micro-expression and phonation) are demonstrated, showing its potential for applications in wearable electronics. This work also offers an alternative route to reuse waste thermosetting resins which would otherwise be difficult to recycle.

\section{Introduction}

Flexible strain sensors are one of the most important components in wearable electronics and have been extensively studied in recent years. ${ }^{[1-5]}$ They exhibit high sensitivity, ${ }^{[6]}$ considerable stretchability, ${ }^{[7]}$ and excellent durability, ${ }^{[8]}$ which makes them widely utilized in sports, personal health monitoring, soft robots, and human-computer interaction. ${ }^{[9-12]}$ To date,

L. Wang, Dr. D. Xiang, X. Zhang, Prof. Y. Li, Y. Zheng, Dr. C. Zhao,

Dr. P. Wang, School of Materials Science and Engineering

Southwest Petroleum University

Chengdu 610500, China

E-mail: dxiang01@hotmail.com; yuntaoli@swpu.edu.cn

Prof. E. Harkin-Jones, School of Engineering

University of Ulster

Jordanstown BT37 OQB, UK

The ORCID identification number(s) for the author(s) of this article can be found under https://doi.org/10.1002/mame.201900492.

DOI: 10.1002/mame.201900492 various flexible strain sensors based on capacitors, ${ }^{[13]}$ field-effect transistors, ${ }^{[14]}$ and piezoresistive materials ${ }^{[15]}$ have been reported. Among them, piezoresistive strain sensors are advantageous due to their relatively simple structures and straightforward preparation methods..$^{[16-18]}$ These types of strain sensors are typically fabricated by introducing conductive fillers into soft elastomer materials. The strain or deformation applied to the sensor can be determined by measuring the change in the electrical resistance.

The most commonly used conductive fillers in these sensors to date have been metallic particles and carbon nanomaterials, including carbon black (CB), ${ }^{[19,20]}$ carbon nanotubes (CNTs), ${ }^{[21,22]}$ and graphene. ${ }^{[23,24]}$ However, metal particles have the disadvantages of chemical instability and requiring relative large loadings, which leads to reduction in processability and in the flexibility of strain sensors. In contrast, carbon nanomaterials with excellent electrical conductivity have been regarded as promising additives for flexible strain sensors. For instance, Pan et al. ${ }^{[25]}$ demonstrated a stretchable strain sensor based on 3D graphene films, which exhibited excellent strain-sensing performance with a workable strain range of up $187 \%$ and simultaneous gauge factor of up to 1500. Although carbon nanomaterials endow flexible strain sensors with superior electromechanical performance, the high costs of raw materials due to complex fabrication has greatly hampered their mass production.

From an environment and economic perspective, conductive carbonized materials based on biomass or industrial waste plastics are attractive choices due to their low cost, availability, and sustainable sourcing. Through a simple pyrolysis treatment, these nonconductive materials transform into carbonized conductive matter, thereby becoming effective fillers for flexible strain sensors. In recent years, biomaterials such as cotton, ${ }^{[26,27]}$ silk, ${ }^{[28]}$ lignin, ${ }^{[29]}$ and bamboo fibers, ${ }^{[30]}$ have been increasingly used to fabricate flexible strain sensors. For instance, highly efficient strain sensors based on carbonized cotton fabric from commercial clothes were prepared by Zhang et al., ${ }^{[31]}$ with a large working strain range $(\varepsilon>140 \%)$, high sensitivity 
$(\mathrm{GF}=64)$, and good mechanical durability over 2000 loading cycles. Similarly, Li et al. ${ }^{[32]}$ prepared a flexible strain sensor by directly using carbonized commercial tissue paper that was recyclable. Most biomass materials have their own particular intrinsic interconnected structures, such as fiber and fabric structures. Therefore, the performance of these strains sensors significantly depends on the source of biomass, which may lead to lower reliability and repeatability. It is widely known that industrial waste plastics, especially waste thermosetting resins, are difficult to recycle due to the hyper-crosslinked polymer chains. Although this structural feature makes thermosetting resins almost infusible and insoluble, it is possible to obtain porous conductive structures after thermosetting resins are carbonized.

Phenol formaldehyde foam (PFF) is a common thermosetting resin that is widely used in heat and sound insulation applications in the construction industry. ${ }^{[33]}$ In this work, we demonstrate the fabrication of a flexible and multipurpose strain sensor by using PFF as a raw material. Commercially purchased PFF was firstly converted into carbonized PFF (cPFF) with tunable electrical conductivity by high-temperature pyrolysis. Then, polydimethylsiloxane (PDMS) was used as the elastomer to encapsulate $\mathrm{CPFF}$ to fabricate a piezoresistive strain sensor. The piezoresistive properties of the PDMS-cPFF multipurpose strain sensor under tension, compression, and three-point bending were studied. The sensor exhibited high sensitivity, stable current response, and excellent durability over many loading cycles. Furthermore, large-scale (finger bending and neck movement) and small-scale (facial micro-expression and phonation) human motion were successfully monitored, thereby demonstrating the potential of the proposed sensor in smart wearable devices.

\section{Experimental Section}

\subsection{Materials}

Commercial phenol formaldehyde foam (PFF) with a volume density of $26 \mathrm{~kg} \mathrm{~m}^{-3}$ was supplied by Xinteda Energy Saving Materials Co., Ltd. (Chengdu). Nitrogen gas with a purity of 99\% was purchased from Sichuan Messer Gas Products Co., Ltd. SYLGARD 184 silicone elastomer kit (Dow Corning, USA) PDMS was used for encapsulation.

\subsection{Preparation}

Figure 1a shows the fabrication procedure of carbonized PFF (cPFF)-based flexible strain sensors. A PFF block was firstly cut into $17 \mathrm{~mm} \times 7 \mathrm{~mm} \times 2 \mathrm{~mm}$ sheets using a blade and then alternately immersed in ethanol and deionized water for $2 \mathrm{~min}$ for cleaning. After being dried at $80{ }^{\circ} \mathrm{C}$ in an air oven for $1 \mathrm{~h}$, PFF sheets were placed in a tubular furnace (Jingke, OTF-1200X), where specimens were carbonized in an inert atmosphere under a nitrogen gas flow of $100 \mathrm{~mL} \mathrm{~min}^{-1}$. The carbonization process is shown in Figure 1b. The carbonization temperature $\left(\mathrm{T}_{c}\right)$ varied from 400 to $900{ }^{\circ} \mathrm{C}$.

In order to ensure proper encapsulation, $\mathrm{CPFF}$ sheets were carefully immersed in the PDMS liquid prepolymer which was prepared by mixing its base and curing agent at a mass fraction of 10:1 in a beaker. This beaker was then placed in a vacuum oven at $-0.08 \mathrm{MPa}$ and room temperature to remove the bubbles in the cPFF and facilitate the effective infusion of PDMS. After $30 \mathrm{~min}$, soaked cPFF sheets were removed from the beaker and cured at $100{ }^{\circ} \mathrm{C}$ for $35 \mathrm{~min}$ to obtain PDMScPFF sheets.

The ends of the PDMS-cPFF sheet were cut off using a blade to expose the inner conductive $\mathrm{CPFF}$ section and to enable the introduction of electrical contacts. Electrodes made of silver paste were attached to these ends to connect external wires and then cured at $80^{\circ} \mathrm{C}$ for $10 \mathrm{~min}$. The distance between two electrodes was fixed at $15 \mathrm{~mm}$. The as-prepared PDMS-cPFF conductive element was then placed in a customized glass mold and encapsulated by PDMS liquid prepolymer. After being cured at $100{ }^{\circ} \mathrm{C}$ for $35 \mathrm{~min}$ in an air oven, the PDMScPFF strain sensor was finally ready.

\subsection{Characterization}

Thermogravimetric analysis (TGA) was performed using a TGA/SDTA851e thermal analyzer (Mettler Toledo) to understand the pyrolysis behavior of PFF in an inert $\mathrm{N}_{2}$ atmosphere. The heating rate was fixed at $20^{\circ} \mathrm{C} \mathrm{min}-1$ and the temperature ranged from 50 to $1000{ }^{\circ} \mathrm{C}$. Morphologies of PFF, cPFF, and PDMS-CPFF as well as their main elemental analysis were studied using scanning electron microscopy with an energydispersive X-ray spectroscope (SEM-EDS, Zeiss EVO MA15).
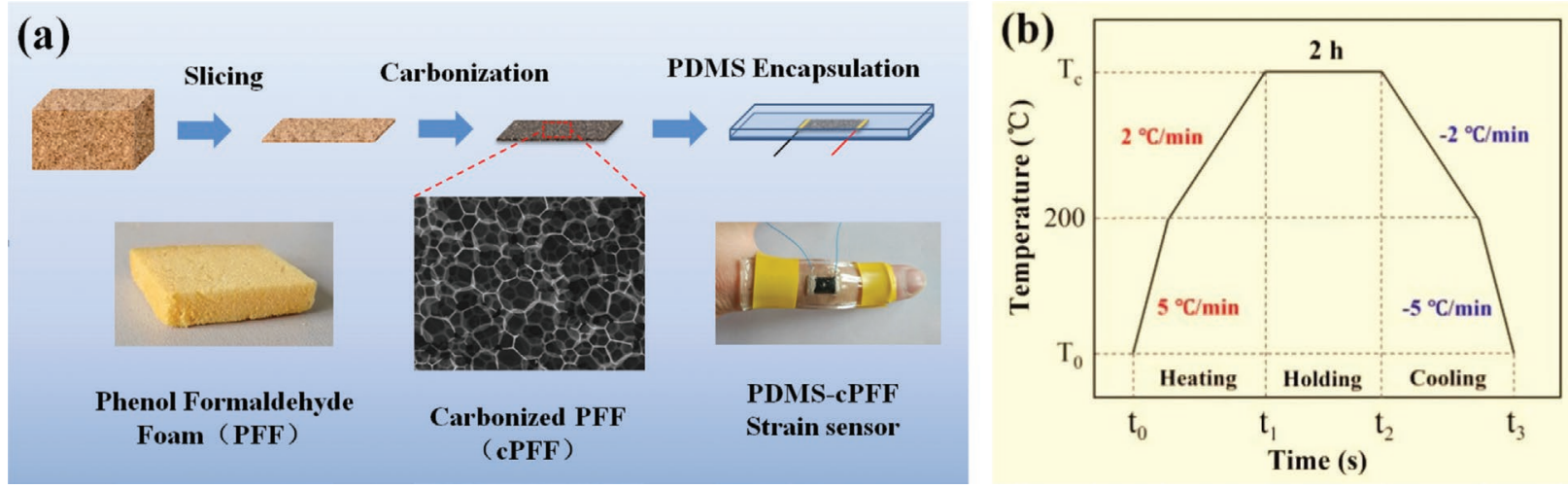

Figure 1. a) Fabrication of the PDMS-cPFF multipurpose strain sensor and b) related carbonization process parameters. 


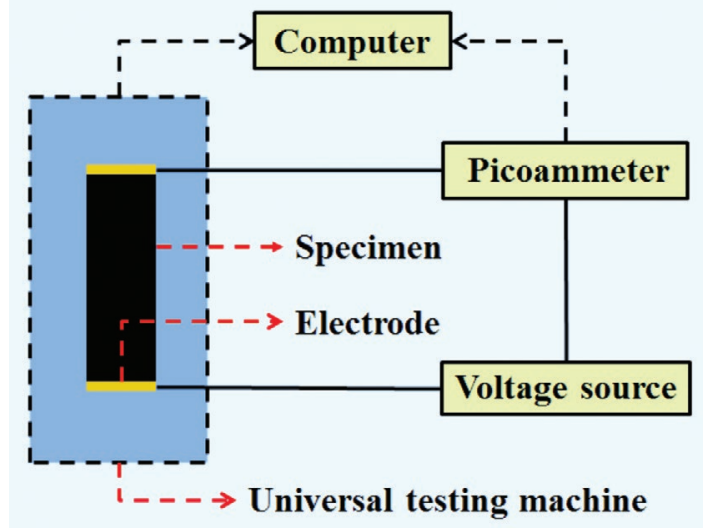

Figure 2. In-house developed electromechanical measuring system.

Prior to imaging, fractured surfaces of specimens were sputtered with gold. Raman spectroscopy was carried out using a micro-Raman spectrometer (IDRaman micro IM-52) with $785 \mathrm{~nm}$ laser excitation and a spectral range of $200-2000 \mathrm{~cm}^{-1}$.

The volume conductivity $\left(\sigma_{V}\right)$ of the PDMS-cPFF conductive elements was measured using a two-point method with a picoammeter (Keithley 6485) and DC voltage source (Tektronix PWS4323), using the formula: $\sigma_{V}=(L \times I) /(U \times S)$, where $L$ and $S$ are the length and cross-sectional area of specimens; $U$ is the output voltage from the source and $I$ is the measured current. To investigate the strain-sensing behavior of the PDMScPFF strain sensor, an in-house developed electromechanical measuring system (Figure 2) was used, which consisted of a universal testing machine (MTS CMT-4101), the above picoammeter, and DC voltage source. The specimens were subject to various forms of loading such as tension, compression, and three-point bending under an applied voltage of $3 \mathrm{~V}$. All electrical and mechanical data were collected using specialized software.

\section{Results and Discussion}

\subsection{Electrical Conductivity and Morphology}

From the TGA curve and its differential values (DTG) of PFF (Figure 3a), it is evident that the maximum degradation temperature $\left(T_{\max 2}\right)$ is about $570{ }^{\circ} \mathrm{C}$, where PFF rapidly converts into carbon. Hence, an initial $T_{c}$ of $400{ }^{\circ} \mathrm{C}$ was chosen to study the electrical conductivity of carbonized PFF. Figure $3 \mathrm{~b}$ shows the effect of $T_{c}$ on the $\sigma_{V}$ of cPFF and on mass residual ratio. When PFF was carbonized at a low $T_{c}$, the $\sigma_{V}$ of the corresponding cPFF also had a low magnitude. For example, $\sigma_{V}$ of cPFF-400 and CPFF-500 are $6.1 \times 10^{-10} \mathrm{~S} \mathrm{~m}^{-1}$ and $1.5 \times 10^{-9} \mathrm{~S} \mathrm{~m}^{-1}$, respectively. When $T_{c}$ was increased to $600{ }^{\circ} \mathrm{C}$, the value of $\sigma_{V}$ significantly increased to $1.3 \times 10^{-5} \mathrm{~S} \mathrm{~m}^{-1}$, which is four orders of magnitude higher than cPFF-500. When $T_{c}$ exceeded $700{ }^{\circ} \mathrm{C}$, the rate of increase of $\sigma_{V}$ decreased but the value reached a relatively high conductivity level. Considering both the optimal electrical conductivity and the Joule heating effect, ${ }^{[34]}$ we choose cPFF-700 $\left(\sigma_{V}=0.8 \mathrm{~S} \mathrm{~m}^{-1}\right)$ as the material for the preparation of the flexible strain sensor.

The SEM images of PFF and CPFF-700 are shown in Figure 4. It can be observed that the pore diameters of both specimens in Figure 4a,b are relatively uniform. The average pore sizes $\left(D_{A}\right)$ of PFF and cPFF-700 as determined using the Nano Measurer software are 179.9 and $178.7 \mu \mathrm{m}$, respectively. Their corresponding pore size distributions are 78.5-298.8 $\mu \mathrm{m}$ and 81.9-287.4 $\mu \mathrm{m}$, as shown in insets of Figure $4 \mathrm{a}, \mathrm{b}$, respectively. This indicates that the carbonization has a minor influence on the original porous structure of PFF and its main porous framework is retained. In addition, compared to PFF (Figure 4a-1), some breakage can be observed in the porous structure of the cPFF-700 (Figure 4b-1), which can be attributed to degradation of the thin cell walls in PFF due to high-temperature carbonization.

Two aspects are considered when investigating the effect of carbonization on the conductivity of cPFF. Firstly, carbonization can change the elemental composition of PFF. From results of EDS (Figure 5a), it can be seen that the main elements in CPFF700 are $\mathrm{C}, \mathrm{O}$, and $\mathrm{P}$ with $93.1,17.0$, and $2.2 \mathrm{wt} \%$ composition, respectively. Compared to PFF, C increases by 14.6 wt\% while O decreases by $13.6 \mathrm{wt} \%$.

Secondly, when PFF is carbonized under a relatively high $T_{c}$, part of the amorphous carbon transforms into graphitic carbon. This process of graphitization greatly improves the electrical conductivity, since the conjugated benzene rings in graphite sheets are beneficial for current conduction. ${ }^{[29]}$ Compared to PFF, the Raman spectrum of cPFF-700 (Figure 5b) shows two distinct peaks, around 1330 and $1597 \mathrm{~cm}^{-1}$, which correspond to the $\mathrm{D}$ band and $\mathrm{G}$ band peaks, respectively. The $\mathrm{G}$ band is associated with microcrystalline $\mathrm{sp}^{2}$ carbon and the $\mathrm{D}$ band is related to its defects. ${ }^{[35]}$ The ratio of their peaks intensities
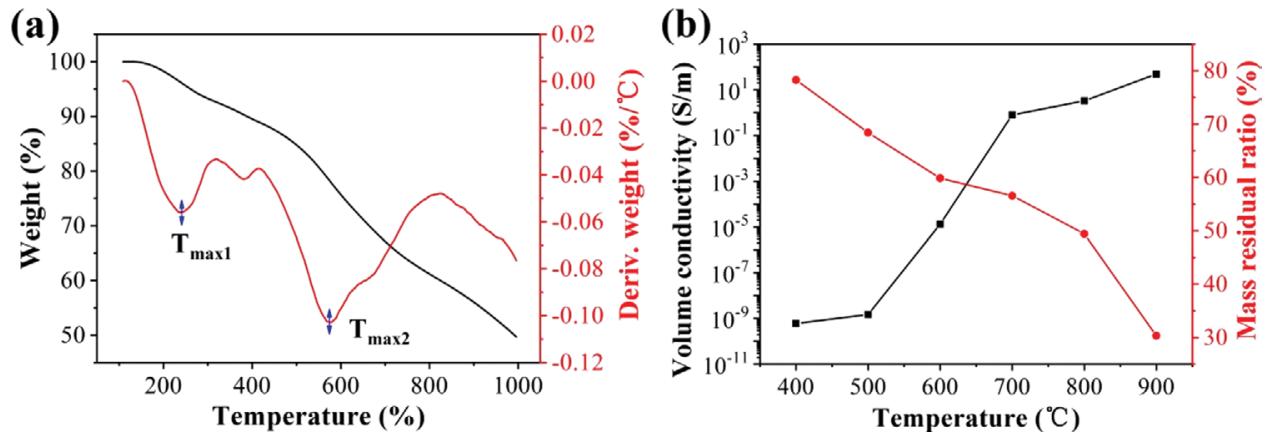

Figure 3. a) TGA and DTC curves of PFF and b) volume conductivity of CPFF under different carbonization temperatures. 

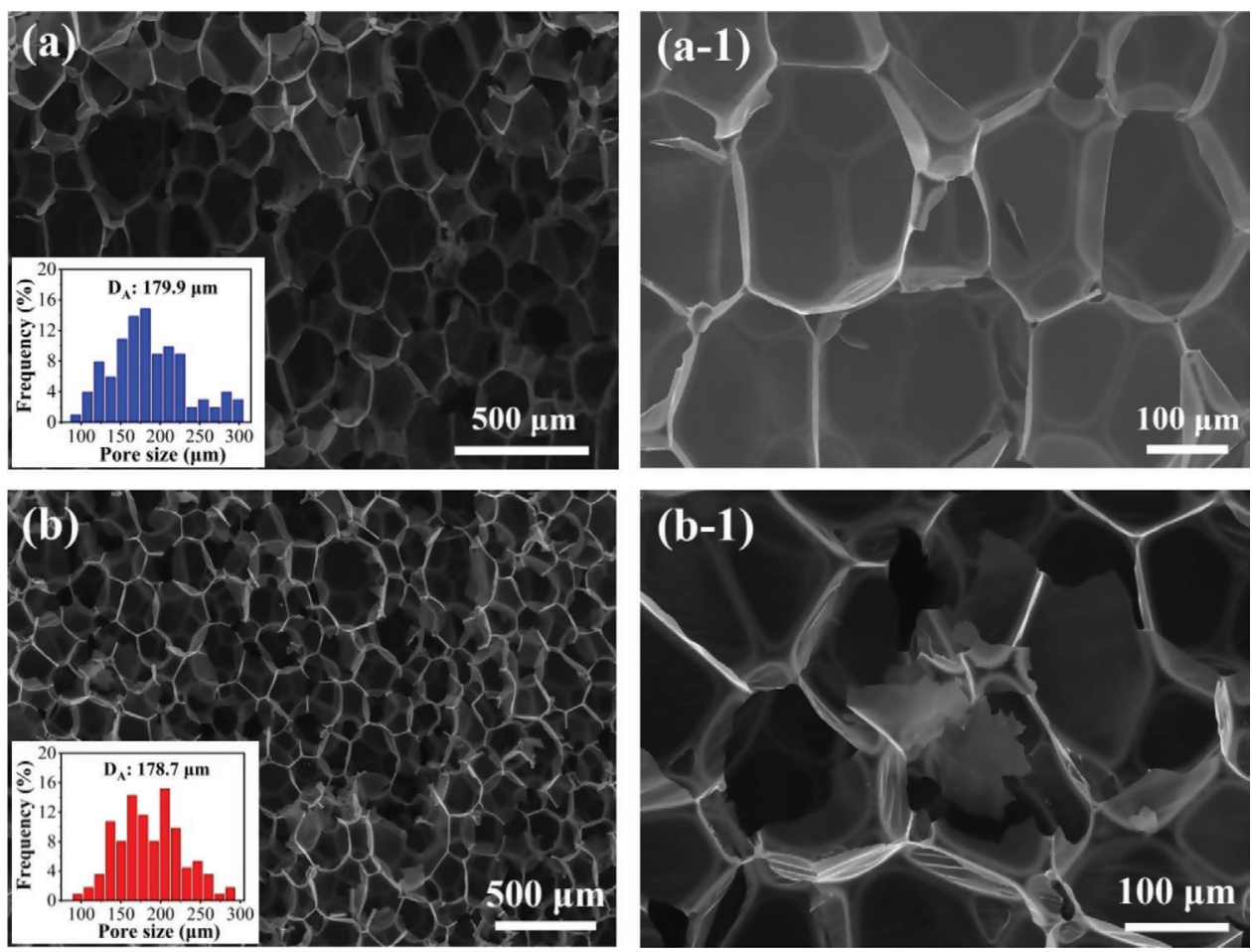

Figure 4. SEM images of a) PFF and b) CPFF-700.

$\left(I_{G} / I_{D}\right)$ are related to the degree of graphitization to some extent. ${ }^{[36]}$ For CPFF-700, the $I_{G} / I_{D}$ value is calculated to be 1.12 , which confirms the formation of graphitic carbon after the carbonization process.

\subsection{Encapsulation and Pre-Strain Treatment}

Due to the brittle nature of $\mathrm{CPFF}$, its porous structure is easily broken under a small deformation. Hence, it cannot be directly used as a flexible strain sensor. However, after being encapsulated by PDMS, the PDMS-cPFF strain sensor can endure repeatable deformation and retain its original porous structure. From the cross-sectional SEM image of the PDMS-cPFF-700 strain sensor (Figure 6a), one can observe that PDMS has effectively filled the pores of the cPFF-700. Distributions of C, $\mathrm{Si}$, and $\mathrm{O}$ elements in PDMS-CPFF-700 are further examined in the EDS color map (Figure 6b-d). Compared to cPFF, a significant increase in the weight fraction of $\mathrm{Si}$ (increased by $42.0 \mathrm{wt} \%$ ) and $\mathrm{O}$ (increased by $7.7 \mathrm{wt} \%$ ) (Table 1) confirms the presence of PDMS in the porous structure of CPFF.

In order to obtain reliable strain-sensing behavior, the PDMS-cPFF-700 strain sensor was subjected to 30 cyclic prestrain treatments under different strain modes, including tension, compression, and three-point bending. An SEM image of the PDMS-cPFF-700 strain sensor (Figure 7a) obtained after pre-strain treatments shows that some parts of the porous carbon structure have been destroyed, resulting in the formation of carbon fragments. However, these fragments are restricted by the surrounding PDMS, forming a
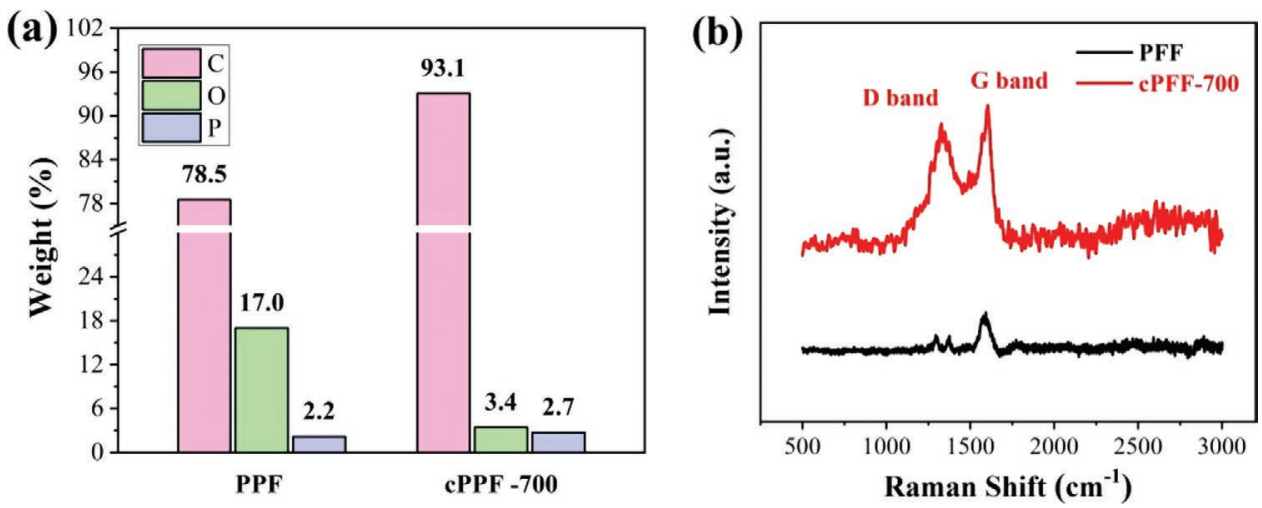

Figure 5. a) Comparison of major element content in PFF and CPFF-700 and b) their Raman spectra. 

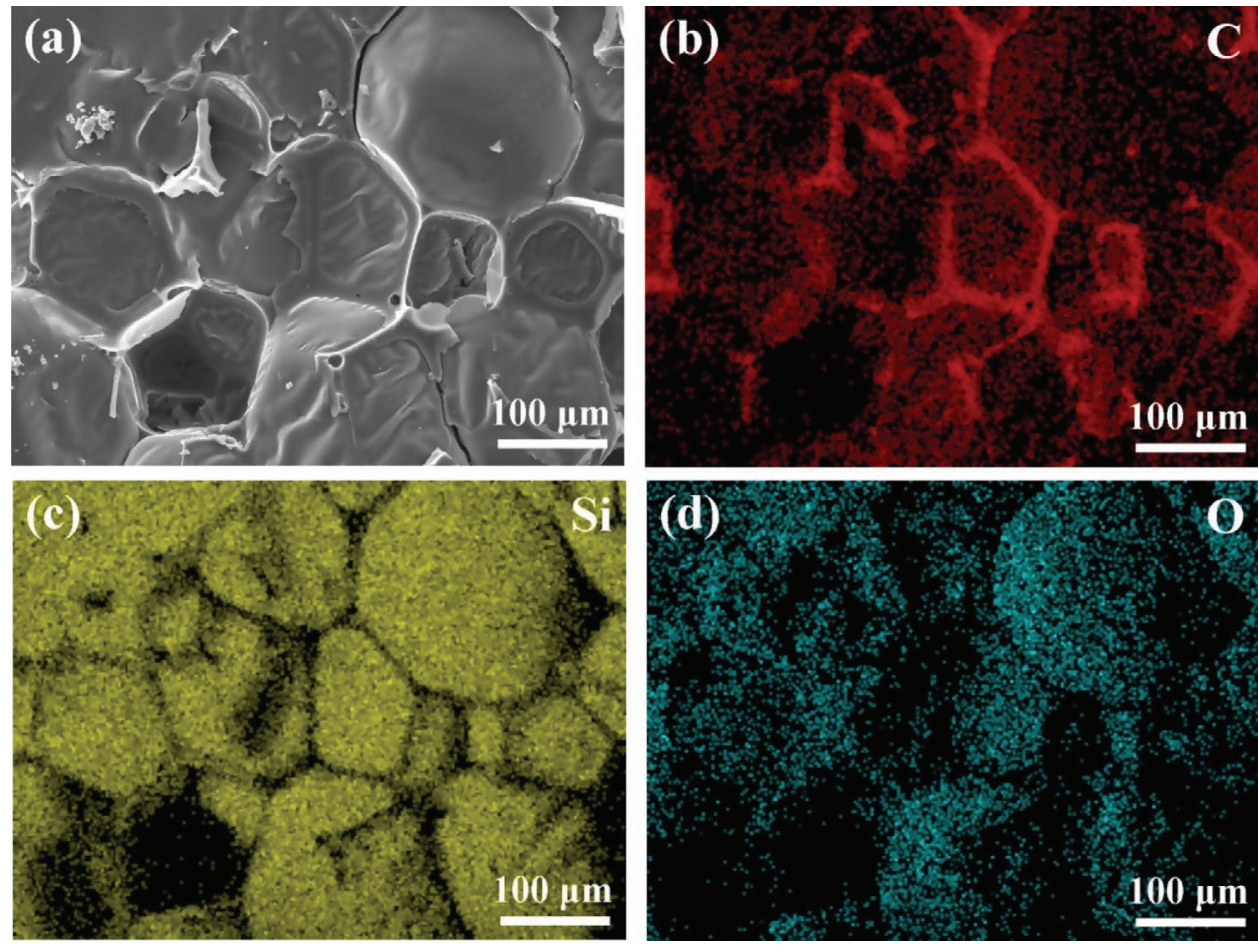

Figure 6. a) Cross-sectional SEM image of PDMS-CPFF-700 strain sensor and b-d) its major elemental distribution from EDS.

so-called fragmented carbon framework (FCF, illustrated in Figure 7b).

Stress-strain curves of the PDMS-cPFF strain sensor under tension, compression, and three-point bending are, respectively, shown in Figure 8a-c. For different pre-strain treatments, their stress-strain hysteresis loop areas are relatively small, indicating that the strains can return to their initial values after the stress is released. For instance, residual strains in 30th cycle of compression (Figure 8b) and three-point bending (Figure $8 \mathrm{c}$ ) are $2.3 \%$ and $0.3 \%$, which indicates this sensor has good elasticity. Furthermore, after 30 cycles of loading, stressstrain hysteresis loops of the PDMS-cPFF strain sensor are almost unchanged, indicating that this strain sensor has a good mechanical repeatability.

\subsection{Piezoresistive Behavior}

Figure 9 shows the strain sensing behavior of the PDMSCPFF-700 strain sensor under different deformations. In order to better characterize the electromechanical performance of strain sensors, the relative electrical current variation $\left(R C V=\Delta I / I_{0}=\right.$ $\left(I-I_{0}\right) / I_{0}$, where $I_{0}$ and $I$ are the initial and real-time current, respectively) was calculated. The relationship between RCV and applied monotonic strain under tension, compression, and

Table 1. Elemental composition of PFF and CPFF-700.

\begin{tabular}{lcccc}
\hline Sample number & $\mathrm{C}[\mathrm{wt} \%]$ & $\mathrm{O}[\mathrm{wt} \%]$ & $\mathrm{P}[\mathrm{wt} \%]$ & Si [wt\%] \\
\hline cPFF-700 & 93.1 & 3.4 & 2.7 & N.A. \\
PDMS-cPFF-700 & 46.9 & 11.1 & N.A. & 42.0 \\
\hline
\end{tabular}

three-point bending are shown in Figure 9a,d,g, respectively. Under tension and three-point bending, $\Delta I / I_{0}$ decreases with the increase of applied strain due to the gradual destruction of conductive network in the sensor, which is shown in Figure S1, Supporting Information. In addition, two linear regions with different slopes are observed in both RCV-strain curves. The slope of the RCV curves reflects the sensitivity of the strain sensors, which is denoted as the gauge factor $(\mathrm{GF}=R C V / \Delta \varepsilon$, where $\Delta \varepsilon$ is the change of applied strain). From Figure $9 \mathrm{a}, \mathrm{g}$, it can be seen that $\mathrm{GF}_{1}(-20.5$ and -19.6$)$ is much lower than $\mathrm{GF}_{2}$ $(-0.3$ and -1.7$)$, indicating that this strain sensor is more sensitive under small strain (e.g., $\Delta \varepsilon<4 \%$ ). Its sensitivity shown in both tension and bending deformation is higher compared with the recently reported studies. ${ }^{[32,37,38]}$ This is probably due to the tunneling effect in the fragmented carbon framework that dominates the conductive mechanism in small strain ranges, ${ }^{[39]}$ leading to large RCV of the strain sensors.

However, as shown in Figure 9d, the RCV-strain relationship is quite different when this sensor is compressed. Obviously, the RCV-strain curve exhibits both negative and positive piezoresistive effects during the entire strain range of $0-40 \%$, where the compressive strain of $20 \%$ is the turning point. To further understand the underlying mechanism, a schematic depicting the change of the internal structure is proposed in Figure 10. When the strain sensor is under small compressive strain, the deformation of PDMS elastomer particles induces a deflection and separation of surrounding carbonized fragments, which can result in electrical disconnection in the previously connected FCF (Figure 10b). Although further compression causes larger deformation of FCF, neighboring conductive fragments can reconnect to generate new conductive pathways (Figure 10c). Therefore, a positive piezoresistive effect 

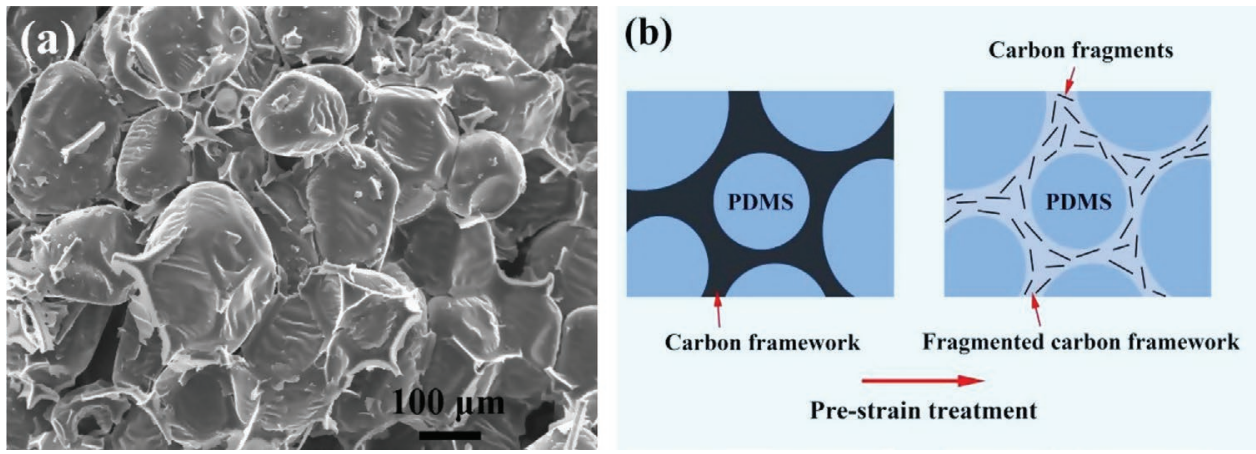

Figure 7. a) SEM image of PDMS-cPFF after pre-strain treatments and b) schematic for the formation of fragmented carbon framework.
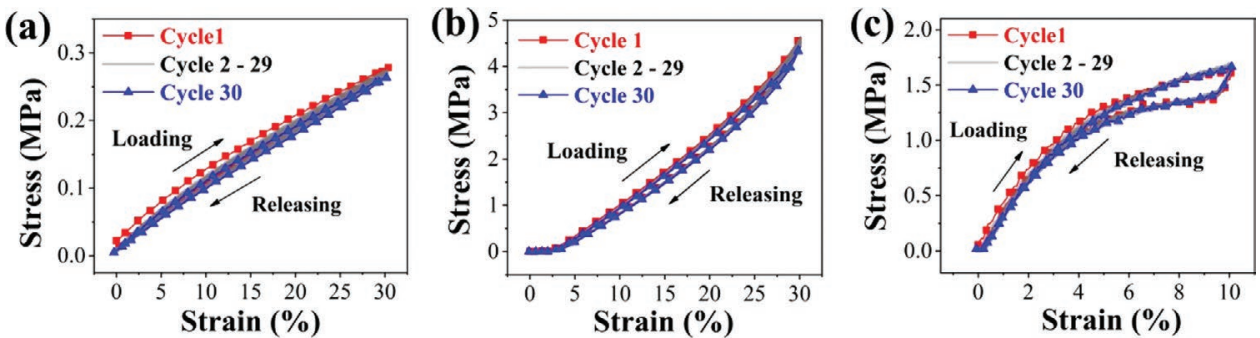

Figure 8. Stress-strain curves of PDMS-cPFF strain sensor under cyclic loading of a) tension, b) compression, and c) three-point bending
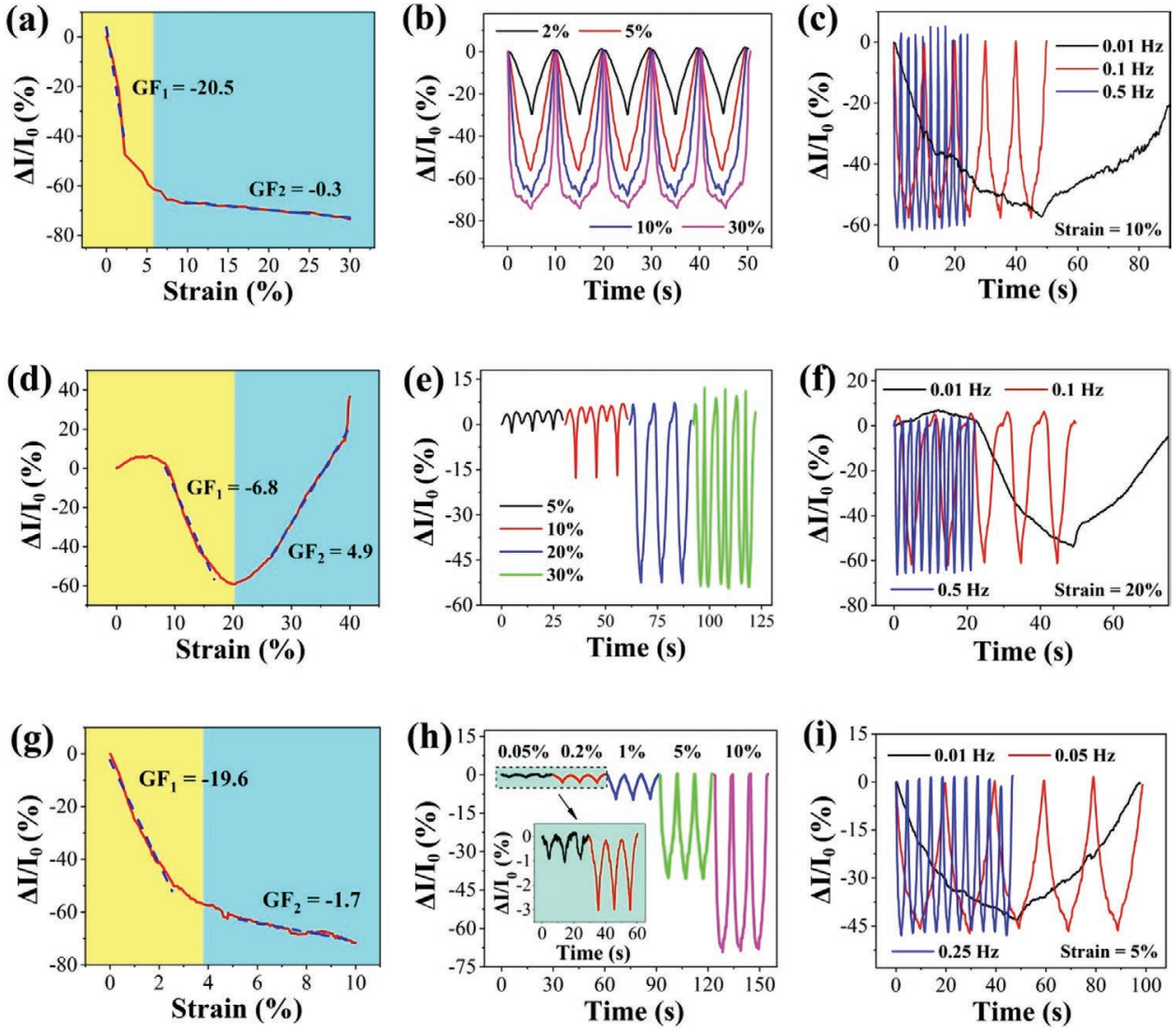

Figure 9. Strain-sensing behaviors of PDMS-cPFF strain sensor under: $a-c)$ tension, $d-f$ ) compression, g-i) three-points bend. 
(a)

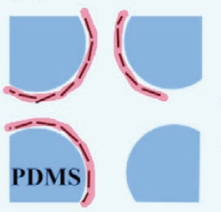

(b)

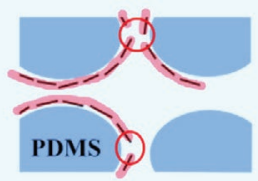

Carbon fragments

Conductive disconnection (c)

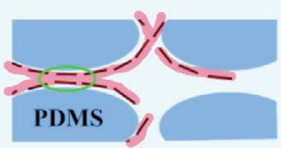

Conductive paths

Conductive reconnection
Figure 10. Schematic of the fragmented carbon framework evolution in the PDMS-cPFF strain sensor: a) before compression, b) under small strain $(<20 \%)$, and c) under large strain $(>20 \%)$.

dominates the strain-sensing behavior when the compressive strain exceeds $20 \%$.

To fully evaluate the piezoresistive properties of the PDMScPFF-700 strain sensor, cyclic loading tests were conducted. RCV responses for different strain ranges at a frequency of $0.1 \mathrm{~Hz}$ were measured, as shown in Figure 9b (tension), Figure 9e (compression), and Figure 9h (three-point bending). For each form of loading, the patterns of each RCV response are identical, indicating the excellent responsiveness of this sensor to applied strains. In addition, the RCV values decrease as the strain increases. For instance, the lower peak values of the PDMS-CPFF-700 strain sensor under tension with 2, 5, 10, and $30 \%$ strain are $-29.5,-59.2,-68.7$, and $-74.2 \%$, respectively, which is consistent with the result shown in Figure 9a. Notably, this sensor can also monitor a subtle applied strain of as low as $0.05 \%$ in three-point bending (Figure $9 \mathrm{~h}$, inset).

The frequency dependence is another important characteristic when piezoresistive properties of a strain sensor are evaluated. RCV responses of the PDMS-cPFF-700 strain sensor under cyclic strain in a frequency range of $0.01-0.5 \mathrm{~Hz}$ were measured. As shown in Figure 9c (tension), Figure of (compression), and Figure 9i (three-point bending), all response signals closely follow the input strains and exhibit almost no frequency dependence. This indicates that the sensor is stable and has a fast response over a wide range of loading frequencies. For example, the minimum RCV values of the PDMS-cPFF-700 strain sensor under the three-point bending at frequencies of $0.01,0.05$, and $0.25 \mathrm{~Hz}$ are $-43.1 \%,-45.7 \%$, and $-46.3 \%$, respectively. Hence, only a decrease of about $3 \%$ is observed.

To demonstrate the durability of this multipurpose strain sensor, cyclic loading/unloading tests under strains of 10, 20, and
$5 \%$ at a frequency of $0.2 \mathrm{~Hz}$ were conducted for tension, compression, and three-points bending, respectively. For the tension mode, the applied voltage was set as $0.6 \mathrm{~V}$. For the compressive and three-points bending modes, the voltage was fixed at $3 \mathrm{~V}$. From Figure 11a-c, it can be observed that the current response signals remain regular even after 1000 cycles, demonstrating the good reliability and excellent durability of this strain sensor.

\subsection{Application}

The high sensitivity and durability of our multipurpose strain sensor make it suitable for monitoring human body motion, including large-scale movements (e.g., bending of joints) and small-scale movements (e.g., facial micro-expression and speaking) (Figure 12). The PDMS-cPFF strain sensor was fixed on the tester's index finger (Figure 12a) and knee (Figure 12b), in order to monitor their bending/releasing movements. The strain sensor deforms when joints are bent, which results in a decrease in its response current. This current value is restored when the joints are relaxed. Similar measurements were performed on monitoring motions of the neck and a significant decrease in current was observed for nodding motion (Figure 12c). Furthermore, some small-scale motions of the human body were also measured by this sensor. As shown in Figure 12d, by attaching the strain sensor on the cheek region of the tester, a real-time current response was observed during smiling movements, which was due to the subtle deformation of the strain sensor induced by facial muscle stretching. In addition, this strain sensor was fixed on the tester's eyelid, which successfully detected small blinking movements (Figure 12e). This strain sensor could also monitor the motion of a human speaking. As shown in Figure 12f, our multipurpose strain sensor was attached to the throat of a tester who was asked to read different English words repeatedly, including monosyllabic word (Hi), dissyllabic word (Sen/sor), and polysyllabic word (Won/der/ful). The response current of the sensor showed a distinctive and repeatable signal pattern for each word, indicating its potential for the application of phonetic recognition.

\section{Conclusions}

In this study, a flexible and multipurpose PDMS-cPFF strain sensor based on carbonized phenol formaldehyde foam was fabricated using a simple and low-cost method. The electrical
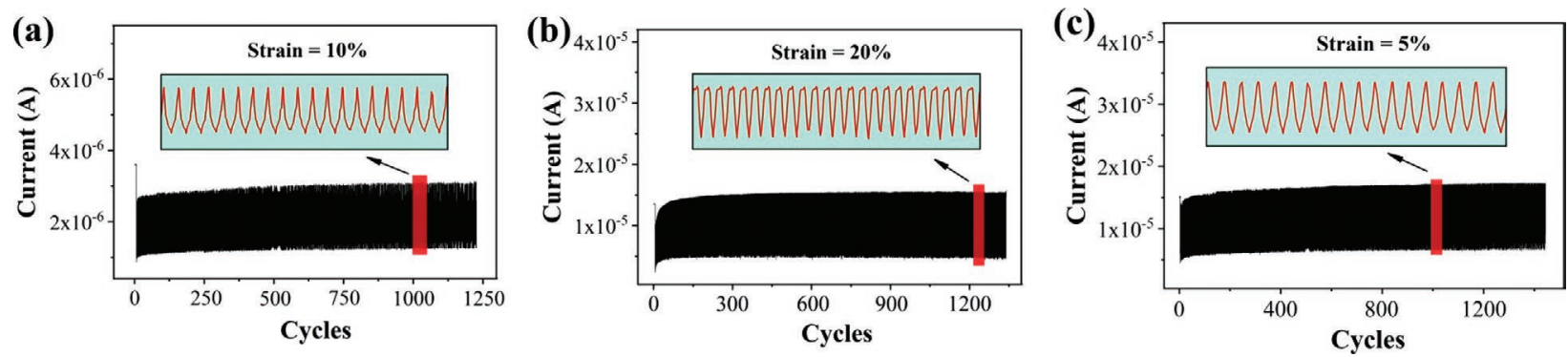

Figure 11. The durability of a PDMS-cPFF strain sensor under cyclic loading of a) tension, b) compression, and c) three-point bending. 

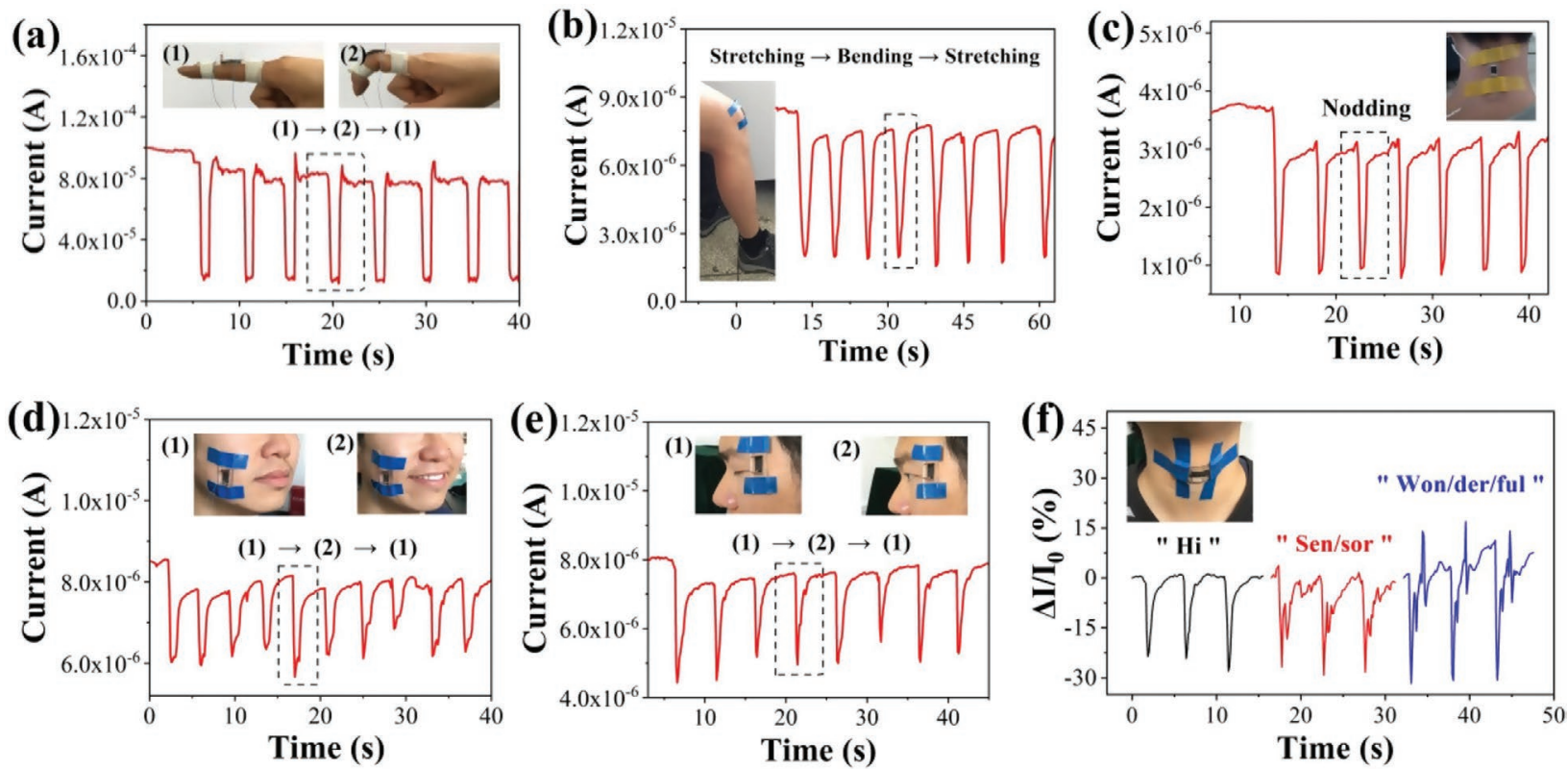

Figure 12. Applications of PDMS-cPFF strain sensor in human motion monitoring: a) index finger and b) knee bending, c) nodding, d) smiling, e) blinking, and f) speaking.

conductivity of cPFF was tuned by varying the carbonization temperature, which influenced the degree of graphitization. The strain sensor was able to monitor various forms of applied strain, including tension, compression, and threepoint bending, and showed an excellent durability over 1000 cycles. Both negative and positive piezoresistive effects of RCV responses were observed during compressive deformation, which was due to the disconnection/reconnection process of conductive carbon fragments. Furthermore, this sensor exhibited high sensitivity $(\mathrm{GF}=-20.5)$ over a strain of $0-5 \%$ in cyclic stretching/releasing tests and was capable of monitoring a subtle strain of $0.05 \%$ in a three-point bending measurement. We also demonstrated its ability to detect both large-scale human body motions (finger bending, nodding) and small-scale motions (facial micro-expression and phonation).

\section{Supporting Information}

Supporting Information is available from the Wiley Online Library or from the author.

\section{Acknowledgements}

All the tests were safe to the participant. An informed, written consent was given by the participant, and all relevant ethical guidelines for human involvement in experiments were adhered to. L.W. and D.X. contributed equally to this work. This work is supported by Sichuan Province Science and Technology Support Program (2017HH0086, 2017jY0152), Undergraduate Innovation Program of Sichuan Province (201910615027), and Scientific Research Foundation for the Returned Overseas Chinese Scholars of Sichuan Province.

\section{Conflict of Interest}

The authors declare no conflict of interest.

\section{Keywords}

carbonization, human motion monitoring, phenol formaldehyde foams, piezoresistivity, strain sensors

Received: July 31, 2019

Revised: September 1, 2019

Published online:

[1] Z. Ma, A. Wei, J. Ma, L. Shao, H. Jiang, D. Dong, Z. Ji, Q. Wang, S. Kang, Nanoscale 2018, 15, 7116.

[2] J. Heo, J. Eom, Y Kim, S. Park, Small 2017, 3, 1703034.

[3] S. Yao, P. Swetha, Y. Zhu, Adv. Healthcare. Mater. 2018, 1, 1700889.

[4] Z. Gu, Y. Xu, C. Lie, R. Fang, Macromol. Mater. Eng. 2018, 10, 1800339.

[5] S. Wang, J. Xu, W. Wang, G. Wang, R. Rastak, F. Molina-Lopez, J. Chung, S. Niu, V. Feig, J. Lopez, T. Lei, S. Kwon, Y. Kim, A. Foudeh, A. Ehrlich, A. Gasperini, Y. Yun, B. Murmann, J. Tok, Z. Bao, Nature 2018, 7694, 83.

[6] Y. Tang, Z. Zhao, H. Hu, Y. Liu, X. Wang, S. Zhou, J. Qiu, ACS Appl. Mater. Interfaces. 2015, 7, 27432.

[7] X. Pu, M. Liu, X. Chen, J. Sun, C. Du, Y. Zhang, J. Zhai, W. Hu, Z. Wang, Sci. Adv. 2017, 5, el700015.

[8] X. Fang, J. Tan, Y. Gao, Y. Lu, F. Xuan, Nanoscale 2017, 45, 17948.

[9] G. Shi, Z. Zhao, J. Pai, I. Lee, L. Zhang, C. Stevenson, K. Ishara, R. Zhang, H. Zhu, J. Ma, Adv. Funct. Mater. 2016, 42, 7614. 
[10] Y. Khan, A. Ostfeld, C. Lochner, A. Pierre, A. Arias, Adv. Mater. 2016, 22,4373

[11] R. Nicolò, B. Blanchet, A. Lamberti, Macromol. Mater. Eng. 2017, 10, 1700161.

[12] M. Amjdi, Y. Yoon, I. Park, Nanotechnology 2015, 37, 375501.

[13] C. Wang, M. Zhang, K. Xia, X. Gong, H. Wang, Z. Yin, B. Guan, Y. Zhang, ACS Appl. Mater. Interfaces. 2017, 15, 13331.

[14] X. Wang, Z. Liu, T. Zhang, Small 2017, 25, 1602790.

[15] W. Obitayo, T. Liu, J. Sensors. 2012, 2012, 652438.

[16] Y. Wang, J. Hao, Z. Huang, G. Zheng, K. Dai, C. Liu, C. Shen, Carbon 2018, 360, 126.

[17] H. Liu, W. Huang, J. Gao, K. Dai, G. Zheng, C. Liu, C. Shen, X. Yan, J. Guo, Z. Guo, Appl. Phys. Lett. 2016, 1, 011904.

[18] C. Bonavolonta, C. Camerlingo, G. Carotenuto, S. Nicola, A. Longo, C. Meola, Sensor Actuat. A-Phys. 2016, 252, 26.

[19] X. Wu, Y. Han, X. Zhang, Z. Zhou, C. Lu, Adv. Funct. Mater. 2016 $34,6246$.

[20] Y. Zheng, Y. Li, Z. Li, Y. Wang, K. Dai, G. Zheng, C. Liu, C. Shen, Compos. Sci. Technol. 2017, 139, 64.

[21] J. Bautista-Quijano, P. Potschke, H. Brunig, G. Heinrich, Polymer 2016, 82, 181.

[22] Q. Chen, D. Xiang, L. Wang, Y. Tang, E. Harkin-Jones, C. Zhao, Compos. Part A. 2018, 112, 186.

[23] C. Boland, U. Khan, C. Backes, A. O'Neill, J. McCauley, S. Duane, ACS Nano 2014, 9, 8819.

[24] Z. Wang, Q. Zhang, Y. Yue, J. Xu, X. Sun, Y. Chen, J. Jiang, Y. Liu, Nanotechnology 2019, 34, 345501.
[25] F. Pan, S. Chen, Y. Li, Z. Tao, J. Ye, K. Ni, Adv. Funct. Mater. 2018, 40, 1803221.

[26] C. Deng, L. Pan, R. Cui, C. Li, J. Qin, J. Mater. Sci.:Mater. Electron. 2017, 4, 3535

[27] J. Ren, C. Wang, X. Zhang, T. Carey, K. Chen, Y. Yin, Carbon 2017, 111, 622.

[28] Q. Wang, M. Jian, C. Wang, Y. Zhang, Adv. Funct. Mater. 2017, 9, 1605657.

[29] B. Wang, T. Shi, Y. Zhang, C. Chen, Q. Li, Y. Fan, J. Mater. Chem. C. 2018, 24, 6423.

[30] A. Afify, S. Ahmad, R. Khushnood, P. Jagdale, J. Tulliani, Sensor Actuat. B. 2017, 239, 1251.

[31] M. Zhang, C. Wang, H. Wang, M. Jian, X. Hao, Y. Zhang, Adv. Funct. Mater. 2016, 2, 104795.

[32] Y. Li, Y. Samad, T. Taha, G. Cai, S. Fu, K. Liao, ACS Sustainable Chem. Eng. 2016, 8, 4288.

[33] C. Mougel, T. Gamier, P. Cassagnau, N. Sintes-Zydowicz. Polymer 2019, 164, 86

[34] R. Zhang, H. Deng, R. Valenca, J. Jin, Q. Fu, E. Bilotti, T. Pejis, Compos. Sci. Technol. 2013, 74, 1.

[35] O. Paris, C. Zollfrank, G. Zickler, Carbon 2005, 1, 53.

[36] T. Yan, Z. Wang, Z. Pan, J Mater. Sci. 2018, 16, 11917.

[37] Y. Li, W. Zhu, X. Yu, P. Huang, S. Fu, N. Hu, K. Liao, ACS Appl. Mater. Interfaces 2016, 48, 33189.

[38] Y. Li, B. Zhou, G. Zheng, X. Liu, T. Li, C. Yan, C. Cheng, K. Dai, C. Liu, C. Shen, Z. Guo, J. Mater. Chem. C. 2018, 6, 2258.

[39] N. Hu, Y. Karube, M. Arai, T. Watanabe, C. Yan, Y. Li, Y. Liu, H. Fukunaga, Carbon 2010, 3, 680. 